\title{
Longitudinal Beam Dynamics for the Heavy-Ion Synchrotron Booster Ring at HIAF
}

\author{
D. Y. Yin (D), J. Liu, G. D. Shen, H. Du (D, J. C. Yang, L. J. Mao, F. C. Cai, and W. P. Chai \\ Institute of Modern Physics, Chinese Academy of Sciences, Lanzhou 730000, Gansu, China \\ Correspondence should be addressed to D. Y. Yin; yindy@impcas.ac.cn
}

Received 24 November 2020; Revised 31 August 2021; Accepted 24 September 2021; Published 20 November 2021

Academic Editor: Sergey Pikuz

Copyright (c) 2021 D. Y. Yin et al. This is an open access article distributed under the Creative Commons Attribution License, which permits unrestricted use, distribution, and reproduction in any medium, provided the original work is properly cited.

To accelerate high-intensity heavy-ion beams to high energy in the booster ring (BRing) at the High-Intensity Heavy-Ion Accelerator Facility (HIAF) project, we take the typical reference particle ${ }^{238} \mathrm{U}^{35+}$, which can be accelerated from an injection energy of $17 \mathrm{MeV} / \mathrm{u}$ to the maximal extraction energy of $830 \mathrm{MeV} / \mathrm{u}$, as an example to study the basic processes of longitudinal beam dynamics, including beam capture, acceleration, and bunch merging. The voltage amplitude, the synchronous phase, and the frequency program of the RF system during the operational cycle were given, and the beam properties such as bunch length, momentum spread, longitudinal beam emittance, and beam loss were derived, firstly. Then, the beam properties under different voltage amplitude and synchronous phase errors were also studied, and the results were compared with the cases without any errors. Next, the beam properties with the injection energy fluctuation were also studied. The tolerances of the RF errors and injection energy fluctuation were dictated based on the CISP simulations. Finally, the effect of space charge at the low injection energy with different beam intensities on longitudinal emittance and beam loss was evaluated.

\section{Introduction}

In China, the Heavy Ion Research Facility at Lanzhou (HIRFL) $[1,2]$ is one major national research facility focusing on nuclear physics, atomic physics, heavy ion applications, and interdisciplinary research. A series of remarkable results have been obtained at HIRFL [3-8]. Based on the effective construction and successful operation of HIRFL [9], a new facility named High-Intensity Heavy-Ion Accelerator Facility (HIAF) [10] has been proposed and designed by the Institute of Modern Physics (IMP), Chinese Academy of Sciences (CAS), in 2009. As indicated in Figure 1, the HIAF comprises a SECR (superconducting electron-cyclotron-resonance ion source) [11], a superconducting iLinac [12], a high-intensity synchrotron BRing (booster ring) [10], a multifunction high-precision synchrotron SRing (spectrometer ring) [13], and a superconducting radioactive beam line HFRS (fragment separator) [14] to connect the two rings. As a more powerful facility, HIAF can provide intense primary and radioactive ion beams for nuclear physics [15-17], plasma physics [18], atomic physics [19], and related research fields.
The BRing, as the key part of the HIAF, has been designed to accumulate and accelerate heavy-ion beams to high intensity and energy with high efficiency, and the important parameters are listed in Table 1. To realize these purposes, it is necessary to control the emittance growth and beam loss to an allowable level of each process including capture, acceleration, and an additional bunch merging. In this paper, we take ${ }^{238} \mathrm{U}^{35+}$, which can be accelerated up to roughly $830 \mathrm{MeV} / \mathrm{u}$ corresponding to the maximum magnetic rigidity of $34 \mathrm{Tm}$, as an example to study the longitudinal beam dynamics by theoretical calculation and numerical simulation. The numerical model is given by a scalable multi-macroparticle simulation platform CISP [20], and 10000 macroparticles are applied during the simulation process. In order to quantify the beam properties during the whole processes, several outputs such as energy, momentum, rms emittance, rms bunch length, space charge voltages, bunching factor, and RF amplitude ramps are plotted as a function of time or revolution turn.

The content is organized as follows: in Section 2, we calculate the basic RF program for beam capture, the beam 


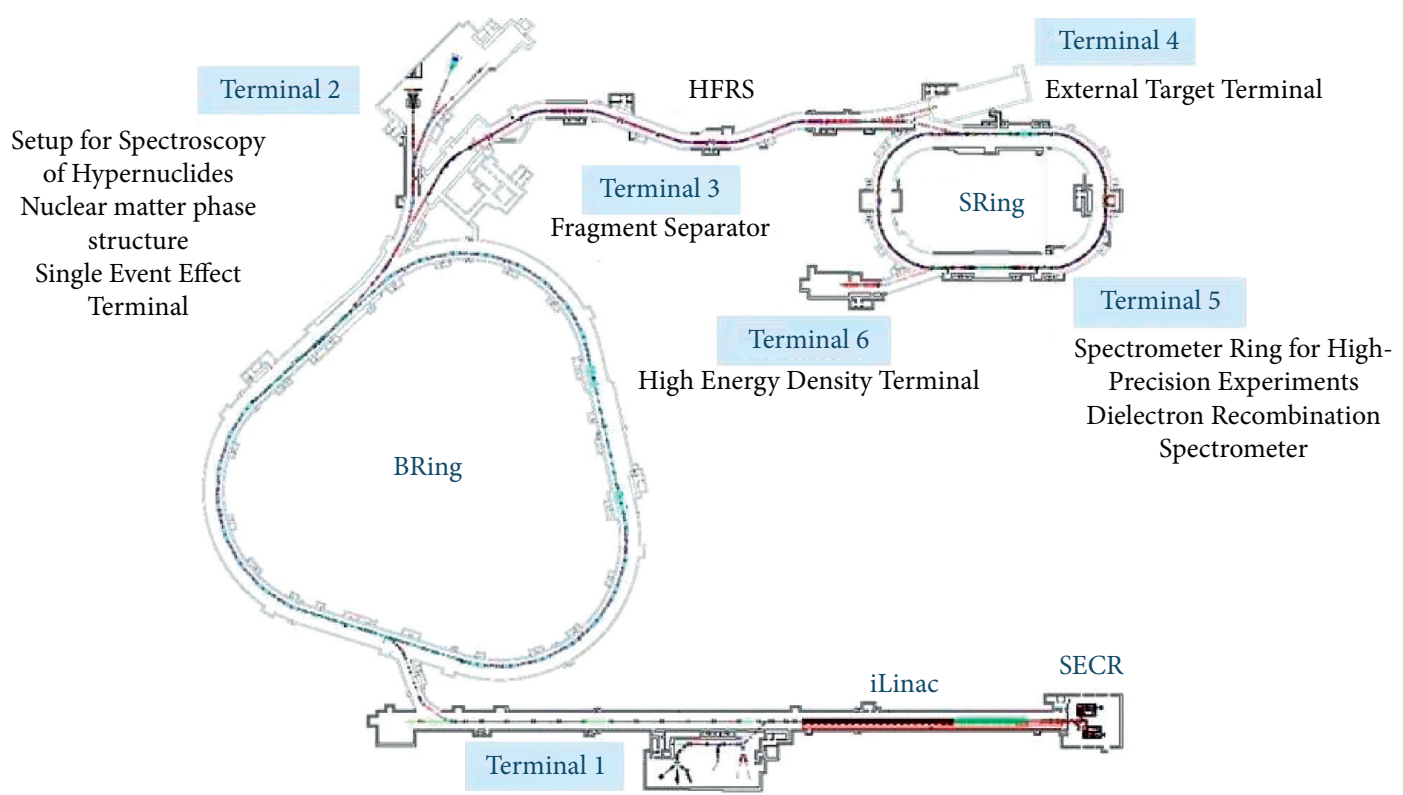

Low Energy Nuclear Structur Spectrometer Intensive Ion Beams Irradiation

Figure 1: General layout of HIAF.

TABLE 1: Main parameters of the BRing.

\begin{tabular}{|c|c|c|}
\hline \multirow{4}{*}{ Main parameters } & Length $(\mathrm{m})$ & 569.0985 \\
\hline & Maximum magnetic rigidity $(\mathrm{Tm})$ & 34.0 \\
\hline & Accelerating rate $(\mathrm{T} / \mathrm{s})$ & 12.0 \\
\hline & Operation cycle (s) & $0.45-10000$ (normal mode $0.45 \mathrm{~s}$, slow extraction mode $4-10000 \mathrm{~s}$ ) \\
\hline \multirow{7}{*}{ Lattice } & Superperiod & 3 \\
\hline & & $Q x / Q y=9.47 / 9.43$ (normal mode) \\
\hline & Tune & $Q x / Q y=9.348 / 9.43$ (slow extraction mode) \\
\hline & & $Q x / Q y=14.47 / 10.43($ proton mode) \\
\hline & Transverse acceptance $(\pi \mathrm{mm} \cdot \mathrm{mrad})$ & Ah/Av: $200 / 100(\triangle P / P= \pm 0.5 \%)$ \\
\hline & Transition factor $y$ & 7.65 (normal mode) \\
\hline & Iransition ractor $\gamma$ & 11.22 (proton mode) \\
\hline \multirow{4}{*}{ Injection } & $\begin{array}{c}\text { Ion } \\
\text { Energy }(\mathrm{MeV} / \mathrm{u})\end{array}$ & $\begin{array}{c}p{ }^{238} \mathrm{U}^{35+} \\
48(p), 17\left({ }^{238} \mathrm{U}^{35+}\right)\end{array}$ \\
\hline & Injection current (emA) & $2.0(p), 1.0\left({ }^{238} \mathrm{U}^{35+}\right)$ \\
\hline & Momentum spread $(\Delta p / p)$ & $\leq \pm 2 \times 10^{-3}$ \\
\hline & Emittance $(\pi \mathrm{mm} \cdot \mathrm{mrad})$ & $\leq 5(6 \sigma)$ \\
\hline \multirow{2}{*}{ Extraction } & Maximum energy $(\mathrm{MeV} / \mathrm{u})$ & $9300(p), 830\left({ }^{238} \mathrm{U}^{35+}\right)$ \\
\hline & Current (ppp) & $2.0 \times 10^{12}(p), 3.0 \times 10^{10}\left({ }^{238} \mathrm{U}^{35+}\right)$ \\
\hline \multirow{7}{*}{ Magnet } & Maximum field of dipoles $(\mathrm{T})$ & 1.58 \\
\hline & Bending radius of dipoles $(\mathrm{m})$ & 22.0 \\
\hline & Maximum field of quadrupoles $(\mathrm{T} / \mathrm{m})$ & 11 (arc), 11.1 (straight) \\
\hline & Field of sextupoles $\left(\mathrm{T} / \mathrm{m}^{2}\right)$ & $\leq 34$ \\
\hline & & $162 \times 94$ (dipoles) \\
\hline & Aperture $\left(\mathrm{mm}^{2}\right)$ & $192 \times 106$ (quadrupoles in the arc) \\
\hline & & $206 \times 118$ (quadrupoles in the straight) \\
\hline \multirow{3}{*}{ Radio frequency } & Frequency range $(\mathrm{MHz})$ & $\begin{array}{c}0.324-1.049(p) \\
0.397-1.789\left({ }^{238} \mathrm{U}^{35+}\right)\end{array}$ \\
\hline & Peak voltage $(\mathrm{kV})$ & 268 \\
\hline & Harmonic number & $h=1,2(p), h=1,2,4\left({ }^{238} \mathrm{U}^{35+}\right)$ \\
\hline
\end{tabular}

ions' distribution evolution in the phase space is simulated, and the beam properties are derived; meanwhile, the space charge effect on longitudinal emittance growth is discussed; in Section 3, the basic RF program for beam acceleration is calculated, and the beam properties are derived by simulations; and in Section 4, the bunch merging processes are studied, and the basic RF program is given. Meanwhile, the beam properties under RF errors and injection energy 
fluctuation are dictated, and the tolerance on these errors based on the emittance growth and beam loss is presented; the results can be found in Appendixes A and B.

After two-plane painting injection and accumulation, the realistic particle distribution of ${ }^{238} \mathrm{U}^{35+}$ with an energy of $17 \mathrm{MeV} / \mathrm{u}$ is shown in Figure 2, a plot, the horizontal axis is circumference $L$ in units of meter $(\mathrm{m})$, and the vertical axis is momentum spread $\Delta p / p$. As can be seen, the beam is completely unbunched, and the rms relative momentum spread is $0.667 \times 10^{-3}$. To accelerate the ${ }^{238} \mathrm{U}^{35+}$ beam from $17 \mathrm{MeV} / \mathrm{u}$ to $830 \mathrm{MeV} / \mathrm{u}$ with minimum longitudinal emittance growth and beam loss, a detailed understanding of the longitudinal beam dynamics during capture and acceleration is necessary. Moreover, in our case, an additional multibunch merging process converting four bunches into one is also needed.

\section{Beam Capture at Injection Energy}

When accelerating the heavy-ion beam in a synchrotron, the bunched beam is needed, which can be accomplished through the capture [21-23]. As one of the potential sources for longitudinal beam quality degradation and beam loss, beam capture requires three principles: (i) the capture must be optimized to keep any beam loss to a minimum to ensure beam intensity and to reduce the radioactive contamination of the machine; (ii) the capture duration should be as short as possible to reduce the machine cycle period, and (iii) the growth of longitudinal emittance $\varepsilon$ at the end of capture should be minimal since the growth of $\varepsilon$ will increase the demands on the RF system. The RF voltage program $V(t)$ [21] for beam capture is determined by the following equation:

$$
V(t)=\frac{V_{i}}{\left(1-t / t_{c} \cdot \sqrt{V_{c}}-\sqrt{V_{i}} / \sqrt{V_{c}}\right)^{2}},
$$

where $V_{i}$ and $V_{c}$ are the initial and final voltage amplitude in the RF cavity, respectively, and $t_{c}$ is the capture time. $V_{i}$, which is the activation value of different electronic circuits in the RF system, is chosen in such a way that the corresponding bucket area generated by it should be much smaller than $\varepsilon$ of the initial injected costing beam. The voltage $V_{c}$ must provide a sufficient bucket area to enclose $\varepsilon$. $t_{c}$ should be large enough compared to the period of the synchronous oscillation to make a linear variation of the phase space parameters, which will help to preserve $\varepsilon$ throughout the capture process.

For the ${ }^{238} \mathrm{U}^{35+}$ beam at the BRing, the revolution frequency is $0.10 \mathrm{MHz}$ at $17 \mathrm{MeV} / \mathrm{u}$, which is far lower than the lower limit of the working frequency of the RF cavity, the RF cavity has to work in the fourth harmonic $(h=4)$ of the revolution frequency according to the design, then, the coasting beam will be captured in four stationary buckets, and four bunches will be generated. In general, the stationary bucket generated by $V_{c}$ according to the following equation (2) [21] should be 1.5 times of $\varepsilon$.

$$
A_{\mathrm{SB}}=16 \sqrt{\frac{e V_{c}}{2 \pi} \frac{\beta^{2} E}{h \omega_{0}{ }^{2} \eta}},
$$

where $e$ is the charge, $\beta=v / c$, in which $v$ is the velocity of particle moving, $E$ is the energy of the synchronous particle, integer $h$ is known as the harmonic number, $\omega_{0}$ is the angular revolution frequency, and $\eta$ is the phase-slip factor. In this case, $\varepsilon$ in the phase space $(\phi, \Delta E / \omega 0)$ is $323 \mathrm{eVs}$, the stationary bucket $A_{\mathrm{SB}}$ should be $485 \mathrm{eVs}$, and the capture voltage $V_{c}$ of $30.29 \mathrm{kV}$ will be required.

First, simulations of the capture, with different $t_{c}$ from $0.005 \mathrm{~s}$ to $0.035 \mathrm{~s}$ with a step of 0.005 at $V_{c}=30.29 \mathrm{kV}$, were performed without any errors of the RF and injection energy fluctuation. In these cases, $V_{i}$ is chosen to be $0.1 \mathrm{kV}$, and the beam ion distributions in the phase space at the end of capture with different $t_{c}$ are shown in Figure 2. The rms momentum spread $\Delta p / p_{\text {rms }}$ and the rms longitudinal emittance $\varepsilon_{\text {rms }}$ [21] are qualitatively derived, which are shown in Figure 3.

As can be seen from Figure $3, \Delta p / p_{\text {rms }}$ is ranged from 0.00149 to 0.00157 , which is very similar to each other; however, $\varepsilon_{\text {rms }}$ decreases from $70.36 \mathrm{eVs}$ to $53.04 \mathrm{eVs}$ as $t_{c}$ increases from $0.005 \mathrm{~s}$ to $0.035 \mathrm{~s}$; among them, $\varepsilon_{\text {rms }}$ of about $55 \mathrm{eVs}$ is almost irrespective of $t_{c}$ when $t_{c} \geq 0.002 \mathrm{~s}$. The maximum $\varepsilon_{\text {rms }}$ of $70.36 \mathrm{eVs}$ occurs when $t_{c}=0.005 \mathrm{~s}$; the reason is the very rapid RF voltage ramping leading to beam filamentation. To balance $\varepsilon_{\text {rms }}$ and the duration of the machine cycle, $t_{c}$ of $0.02 \mathrm{~s}$ will be applied in the capture process.

Apart from $t_{c}, V_{i}$ is also a critical parameter to be controlled. Simulations were also performed for $V_{i}$ from $0.08 \mathrm{kV}$ to $0.14 \mathrm{kV}$ with a step of 0.01 at $V_{c}=30.29 \mathrm{kV}$ and $t_{c}=0.02 \mathrm{~s}$, and $\varepsilon_{\mathrm{rms}}$ are derived which are shown in Figure 4 . The minimum $\varepsilon_{\mathrm{rms}}$ occurs when $V_{i}=0.11 \mathrm{kV}$.

If $V_{c}$ is below $30.29 \mathrm{kV}$, the generated stationary bucket area cannot constrain the injected beam completely, and the beam loss will be inevitable. On the contrary, $V_{c}$ higher than $30.29 \mathrm{kV}$ will induce large momentum spread which may exceed the limited momentum acceptance of the BRing, and the additional beam loss may occur. After a series of calculation, simulation, and optimization, $V_{i}=0.11 \mathrm{kV}$, $V_{c}=30.29 \mathrm{kV}$, and $t_{c}=0.02$ are determined for ${ }^{238} \mathrm{U}^{35+}$ beam capture. $\Delta p / p_{\text {rms }}$ of the captured beam is 0.0015 , and almost all particles can be captured after 1984 machine turns.

For the ${ }^{238} \mathrm{U}^{35+}$ beam, the designed intensity will exceed $3.0 \times 1010$. Such high beam intensity in the BRing is expected to cause an additional collective effect. As one of the important high beam intensity effects, the space charge $[21,24,25]$ plays an important role for the beam dynamics especially. The equation of the longitudinal phase space motion indicates the dependence of particle distribution on the time variations of the RF field, and this external RF field, or voltage, is modified by the field due to the space charge, which consequently modifies the particle distribution and eventually leads to the beam loss or beam quality degradation. 


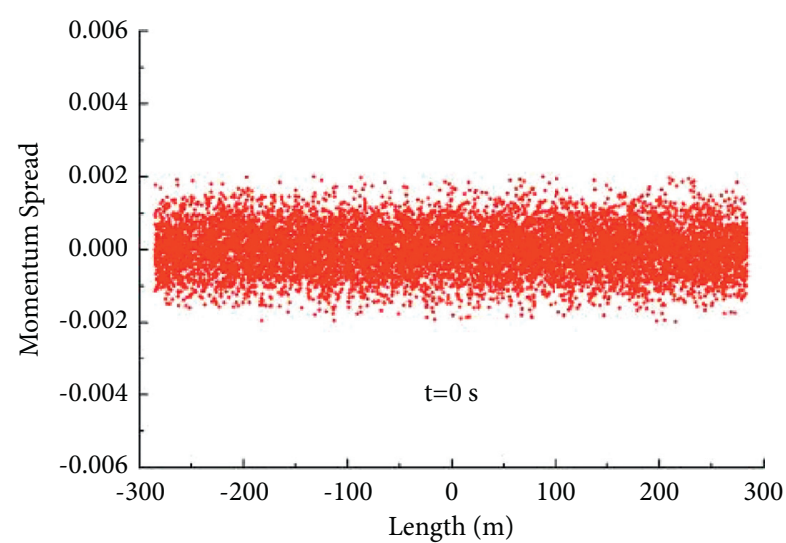

(a)

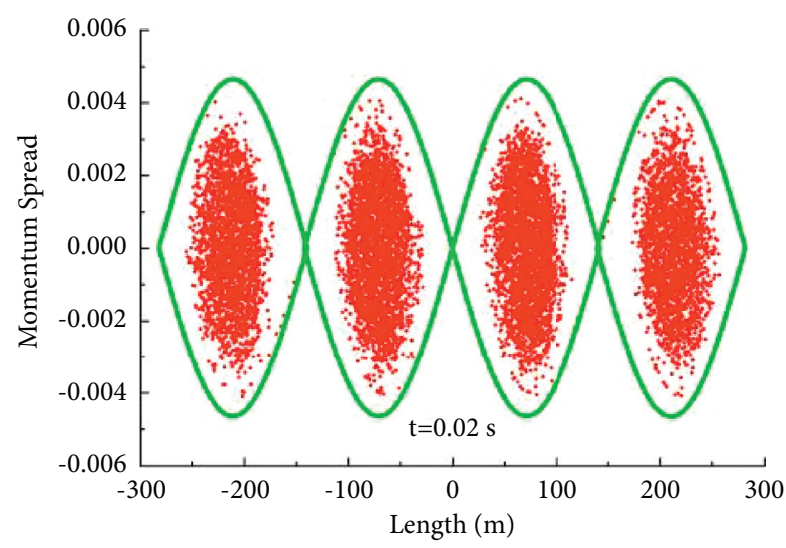

(c)

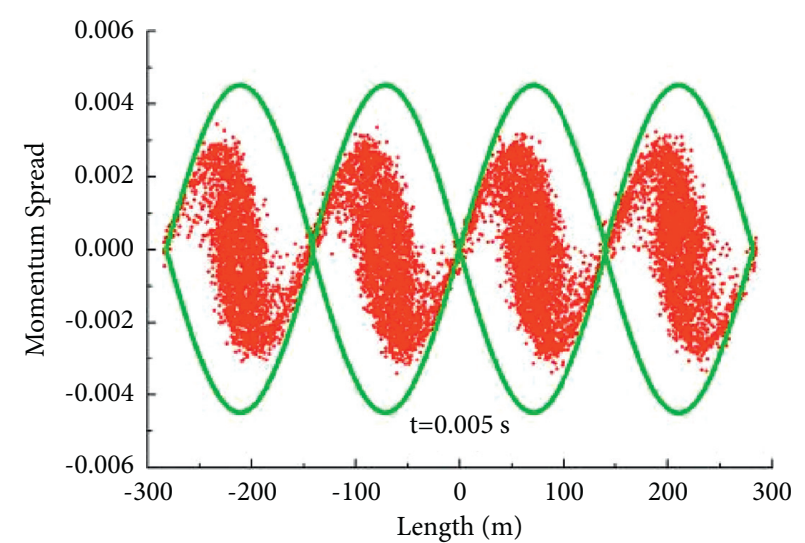

(b)

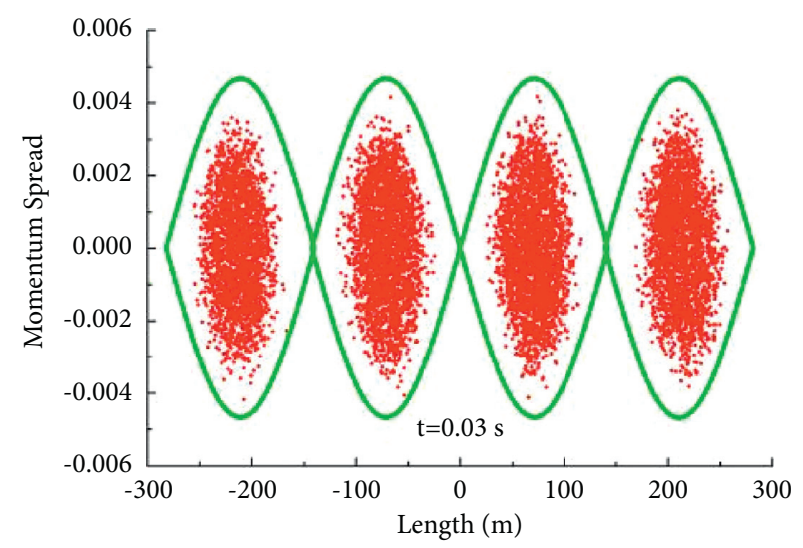

(d)

FIgUre 2: Beam ion distributions in the phase space before (a) and after capture with $t_{c}=0.005 \mathrm{~s}(\mathrm{~b}), 0.02 \mathrm{~s}(\mathrm{c})$, and $0.03 \mathrm{~s}$ (d). In all cases, the $\mathrm{RF}$ voltage is ramped from $V_{i}=0.1 \mathrm{kV}$ to $V_{c}=30.29 \mathrm{kV}$. The bucket separatrix is drawn in green.

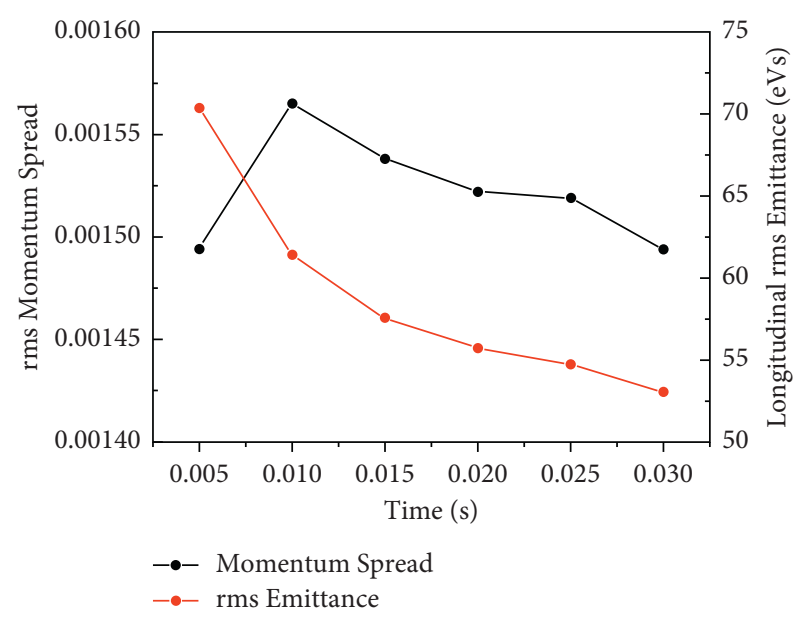

FIGURE 3: $\Delta p / p_{\text {rms }}$ (black) and $\varepsilon_{\text {rms }}($ red) at the end of capture with $t_{c}=0.005 \mathrm{~s}, 0.01 \mathrm{~s}, 0.015 \mathrm{~s}, 0.02 \mathrm{~s}, 0.025 \mathrm{~s}, 0.03 \mathrm{~s}$, and $0.035 \mathrm{~s}$ at $V_{c}=30.29 \mathrm{kV}$.

The space charge is an important issue only in low- and medium-energy accelerators because the space charge voltage per turn scales to $1 / \beta \gamma^{2}$. In this paper, the space charge effect with various beam intensities $N$ ranging from $3.0 \times 1010$ to $1.0 \times 1011$ at the end of capture was estimated, and $\Delta p / p_{\text {rms }}$ and $\varepsilon_{\text {rms }}$ are derived, which are shown in Figure 5 .

It is concluded that the maximum $\varepsilon_{\text {rms }}$ of $56.10 \mathrm{eVs}$ occurs when $N=7.0 \times 1010$, which is increased only by $1.71 \%$ than the case of $N=3.0 \times 1010$. So, the space charge effect on the emittance growth is insignificant if the beam intensity is less than $1.0 \times 1011$.

\section{Beam Acceleration}

After capture, the stationary buckets will be transformed into moving ones to start acceleration through synchronous phase shifting [21, 26, 27]. Since the ionization cross sections and space charge effect decrease largely with the increase of beam energy, the injected low-energy heavy-ion beam should be accelerated to high energy as fast as possible to minimize the significant ionization beam loss, stabilize the dynamic residual gas pressure, and suppress the strong space charge effect; then, a rapid acceleration with a maximum bend magnetic field ramping rate over time $\dot{B}$ of $12 \mathrm{~T} / \mathrm{s}$ is proposed.

During the beam acceleration process, the moving bucket area which is determined by equation (3) should remain approximately invariant of around $485 \mathrm{eVs}$ in $(\phi$, $\Delta E / \omega 0)$. The required voltage amplitude $V_{s}$ and 


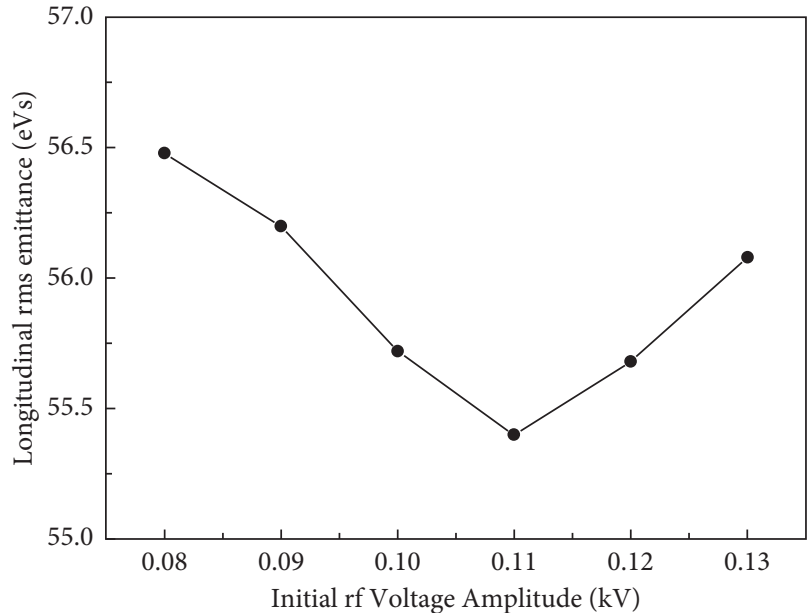

FIgURE 4: $\varepsilon_{\text {rms }}$ at the end of capture with $V_{i}=0.08 \mathrm{kV}, 0.09 \mathrm{kV}$, $0.1 \mathrm{kV}, \quad 0.11 \mathrm{kV}, \quad 0.12 \mathrm{kV}$, and $0.13 \mathrm{kV}$ at $V_{c}=30.29 \mathrm{kV}$ and $t_{c}=0.02 \mathrm{~s}$.

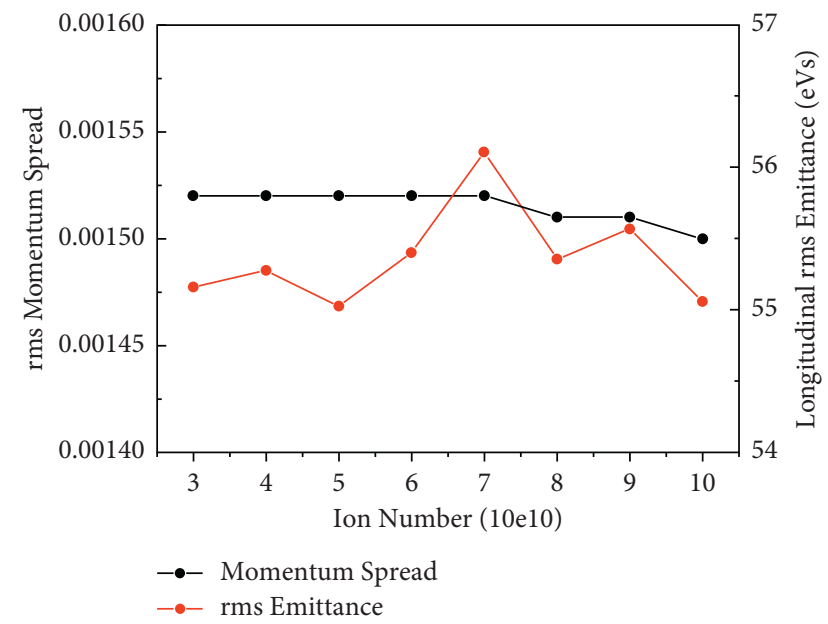

Figure 5: $\Delta p / p_{\text {rms }}$ (black) and $\varepsilon_{\text {rms }}$ (red) with different $N=3.0 \times 1010, \quad 4.0 \times 1010, \quad 5.0 \times 1010, \quad 6.0 \times 1010, \quad 7.0 \times 1010$, $8.0 \times 1010,9.0 \times 1010$, and $1.0 \times 1011$.

synchronous phases $\varphi_{s}$ for the RF acceleration system can be calculated with the following equations (3) and (4) [21]:

$$
\begin{aligned}
A_{\mathrm{AB}} & =16 \sqrt{\frac{e V_{s}}{2 \pi} \frac{\beta^{2} E}{h \omega_{0}^{2} \eta}}\left(\frac{1-\sin \varphi_{s}}{1+\sin \varphi_{s}}\right), \\
\varphi_{s} & =\sin ^{-1} \frac{V_{s}}{V_{m}},
\end{aligned}
$$

in which $V_{m}=L \cdot \rho \cdot \dot{B}_{\max }$ is the voltage that is used to keep the synchronous particle on its ideal orbit, $L$ is the circumference of the BRing, $\rho$ is the bending radius of the dipole magnet, $\dot{B}$ is the ramping rate of the dipole magnetic field over time, $\dot{B}_{\max }$ is the maximum value of $\dot{B}$ available, $V_{s}$ and $\varphi_{s}$ are the needed voltages and synchronous phases, respectively, and $E$ is the particle kinetic energy which is $B$ dependent. The acceleration cycle will be divided into three stages based on $\dot{B}$. The first stage is $\dot{B}$ increasing from $0 \mathrm{~T} / \mathrm{s}$ to the maximum value of $12 \mathrm{~T} / \mathrm{s}$ in $0.05 \mathrm{~s}$, which is determined by hardware equipment such as the magnet and power supply. The second stage is the process of $B$ increasing with constant $\dot{B}$ of $12 \mathrm{~T} / \mathrm{s}$. The third stage is $B$ increasing to the required value while $\dot{B}$ decreasing from $12 \mathrm{~T} / \mathrm{s}$ to $0 \mathrm{~T} / \mathrm{s}$ in $0.05 \mathrm{~s}$. After the three stages, $B$ will increase from $0.18 \mathrm{~T}$ corresponding to the injection energy of $17 \mathrm{MeV} / \mathrm{u}$ to $1.58 \mathrm{~T}$ corresponding to the extraction energy of $830 \mathrm{MeV} / \mathrm{u}$. The programmed $\dot{B}$ (green solid line) and $B$ (green short dot line) curves are shown in Figure 6. Similarly, $V_{s}$ and $\varphi_{s}$ are divided into three stages. At the first stage, $V_{s}$ increases from $30.29 \mathrm{kV}$ up to its maximum amplitude of $268.00 \mathrm{kV}$, and $\varphi_{s}$ increases from $0 \mathrm{rad}$ to $0.58 \mathrm{rad}$, and in this stage, $E$ rises from $17 \mathrm{MeV} / \mathrm{u}$ to $108.87 \mathrm{MeV} / \mathrm{u}$. At the second stage, $V_{s}$ drops from $268.00 \mathrm{kV}$ to $212.24 \mathrm{kV}$, and $\varphi_{s}$ continues to increase from $0.58 \mathrm{rad}$ to $0.764 \mathrm{rad}$, and in this stage, $E$ rises from $108.87 \mathrm{MeV} / \mathrm{u}$ to $595.91 \mathrm{MeV} / \mathrm{u}$. At the last stage, $V_{s}$ continues to drop from $212.24 \mathrm{kV}$ to $4.52 \mathrm{kV}$, and $\varphi_{s}$ drops from $0.764 \mathrm{rad}$ to $0 \mathrm{rad}$, and in this stage, $E$ rises from $595.91 \mathrm{MeV} / \mathrm{u}$ to $830 \mathrm{MeV} / \mathrm{u}$, and the acceleration efficiency is nearly $99 \%$.

\section{Multiple Bunch Merging at the Extraction Energy Platform}

After acceleration, a 4:2 and 2:1 two-step bunch merging $[28,29]$ process will be used to transform four bunches into one to meet the extraction requirement. The first step is to merge four bunches into two bunches; during this step, the voltage amplitude of one RF cavity operating at $h=4$ and the frequency $f_{1}=1.79 \mathrm{MHz}$ is decreased linearly from $V_{1}=4.52 \mathrm{kV}$ to $0.10 \mathrm{kV}$ over merging time $t$; concurrently, the voltage amplitude of another RF cavity operating at $h=2$ and the frequency $f_{2}=0.895 \mathrm{MHz}$ is increased linearly from $0.10 \mathrm{kV}$ to its final value $V_{2} . t$ and $V_{2}$ will affect the longitudinal emittance $\varepsilon$ of the merged bunches, and it will inevitably further affect the following $2: 1$ bunch merging. The simulations, with different $t$ from $0.005 \mathrm{~s}$ to $0.035 \mathrm{~s}$ with a step of 0.005 at different $V_{2}=1 \mathrm{kV}, 2 \mathrm{kV}$, and $3 \mathrm{kV}$, are performed, and $\varepsilon_{\text {rms }}$ and beam loss are derived, which are shown in Figure $7(\mathrm{a})$. The second step is to merge two bunches into one. In this step, $V_{2}$ is decreased linearly from $2 \mathrm{kV}$ to $0.10 \mathrm{kV}$ over time $t_{2}$; meanwhile, the voltage of the $\mathrm{RF}$ cavity working at $h=1$ is increased linearly from $0.10 \mathrm{kV}$ to $V_{3}$. The $\varepsilon_{\text {rms }}$ dependence on $t_{2}$ ranging from $0.005 \mathrm{~s}$ to $0.035 \mathrm{~s}$ and with different $V_{3}=1 \mathrm{kV}, 2 \mathrm{kV}$, and $3 \mathrm{kV}$ is derived, which is shown in Figure $7(\mathrm{~b})$.

According to the $4: 2$ bunch merging simulation result, it was found that $\varepsilon_{\text {rms }}$ tends to decrease with the increase of $t$; however, when $t$ is greater than $0.025 \mathrm{~s}, \varepsilon_{\text {rms }}$ tends to be fixed at around $60 \mathrm{eVs}$. So, the RF program of $t=0.025 \mathrm{~s}$ and $V_{2}=2 \mathrm{kV}$ for the $4: 2$ bunch merging step is determined, the phase $\varphi_{s}$ is fixed at $-1.571 \mathrm{rad}$, the beam distribution in the longitudinal phase space is shown in Figure 8(a), and $\varepsilon_{\mathrm{rms}}$ is $60.19 \mathrm{eVs}$.

For the $2: 1$ bunch merging simulation result, it can be known that $\varepsilon_{\text {rms }}$ decreases with the increase of $t_{2}$, and similar results are obtained with $V_{3}=2 \mathrm{kV}$ and $3 \mathrm{kV}$. However, $\varepsilon_{\text {rms }}$ 


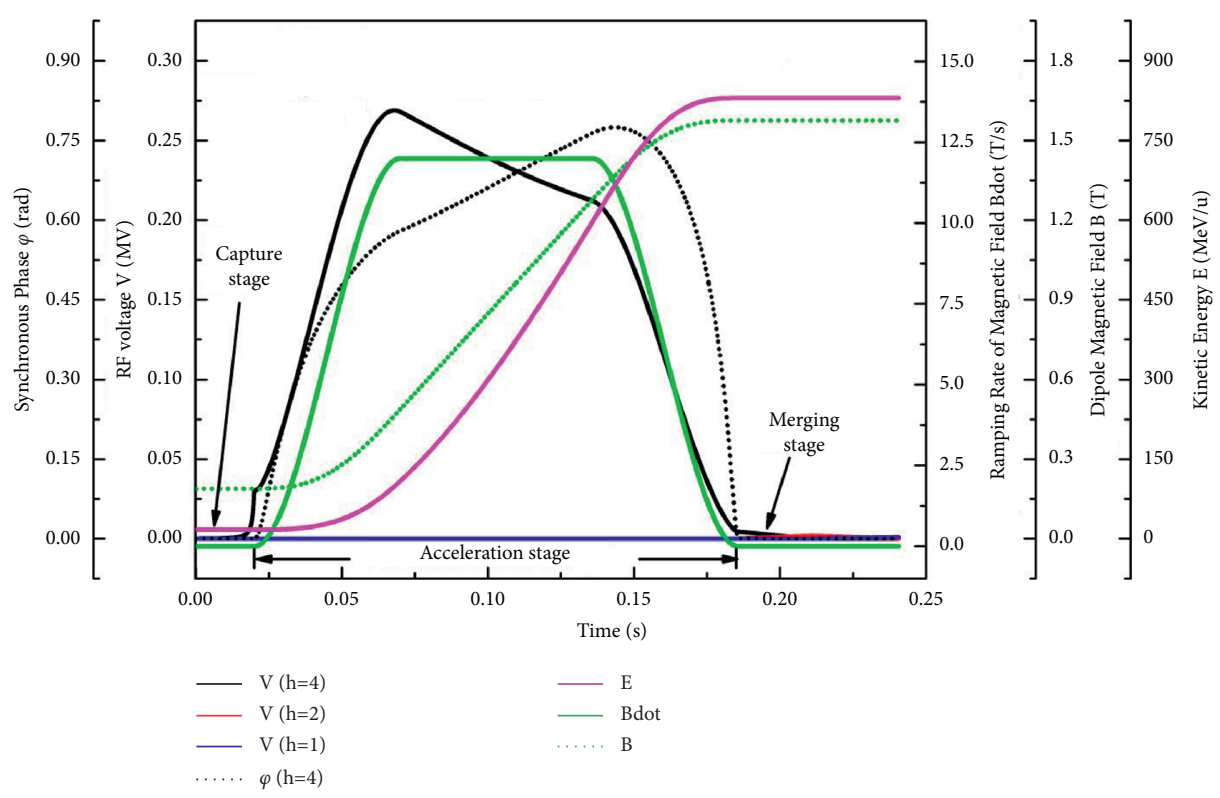

Figure 6: RF voltage programs $V$ and $\varphi_{s}$ of the RF cavity at different operating frequencies, $B, \dot{B}$, and $E$ during the whole process from capture to bunch merging. Black solid line and black short dash dot line are $V_{s}$ and $\varphi_{s}$ of the main $\mathrm{RF}$ cavity with $f=1.79 \mathrm{MHz}(h=4)$, respectively. Red solid line and blue solid line are $V_{2}$ and $V_{3}$, respectively. Beam capture: $0-0.02 \mathrm{~s}$; acceleration: $0.02-0.1856 \mathrm{~s}$; the $4: 2$ bunch merging: $0.1856-0.2106 \mathrm{~s}$; the $2: 1$ bunch merging: $0.2106-0.2406 \mathrm{~s}$.

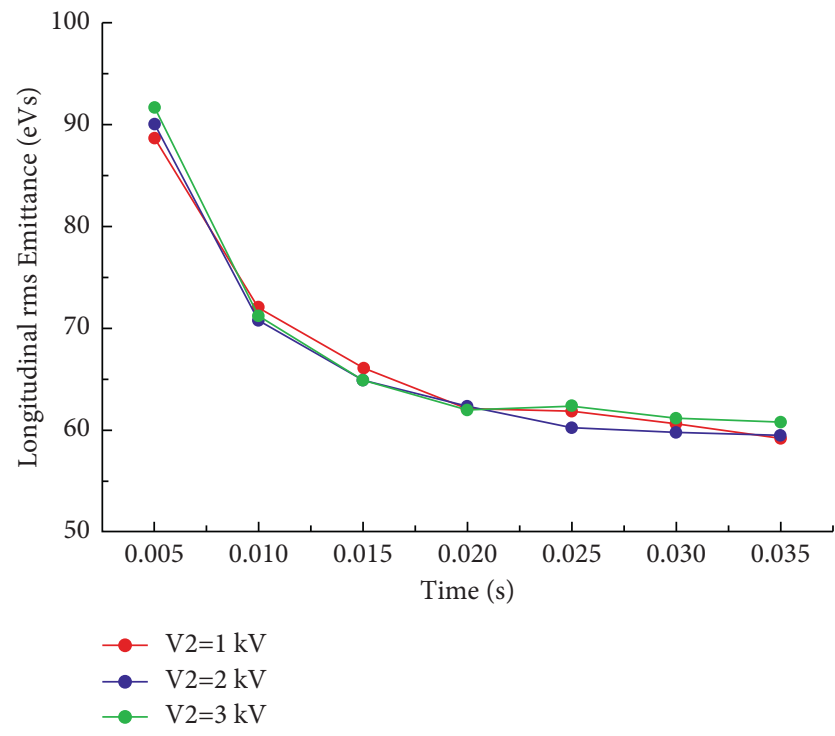

(a)

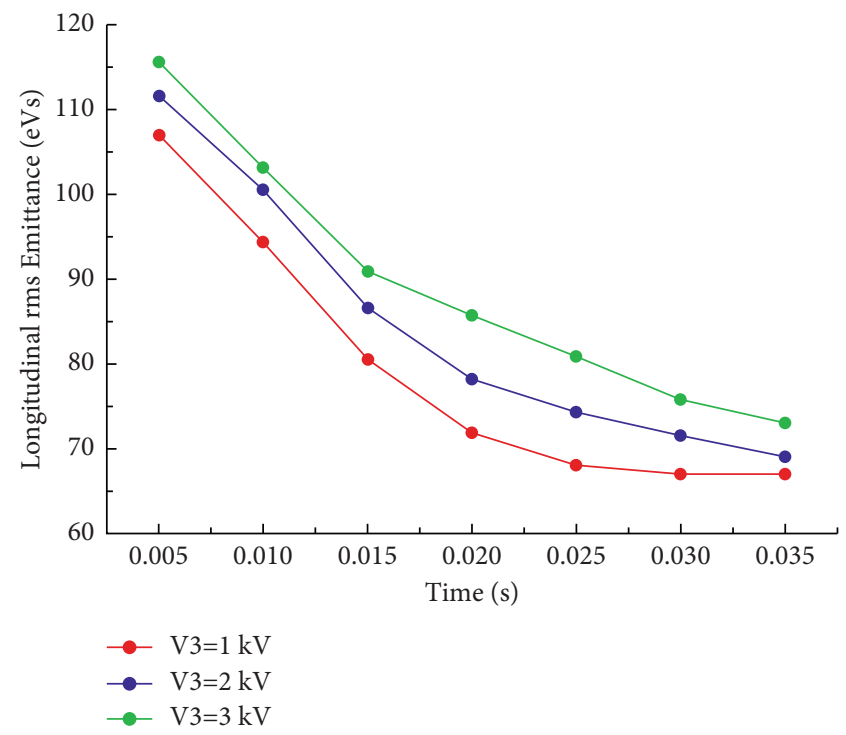

(b)

FIGURE 7: $\varepsilon_{\text {rms }}$ with different merging times at $V_{2}=1 \mathrm{kV}$ (red), $2 \mathrm{kV}$ (blue), and $3 \mathrm{kV}$ (green) at the end of $4: 2$ bunch merging (a) and $2: 1$ bunch merging (b). 


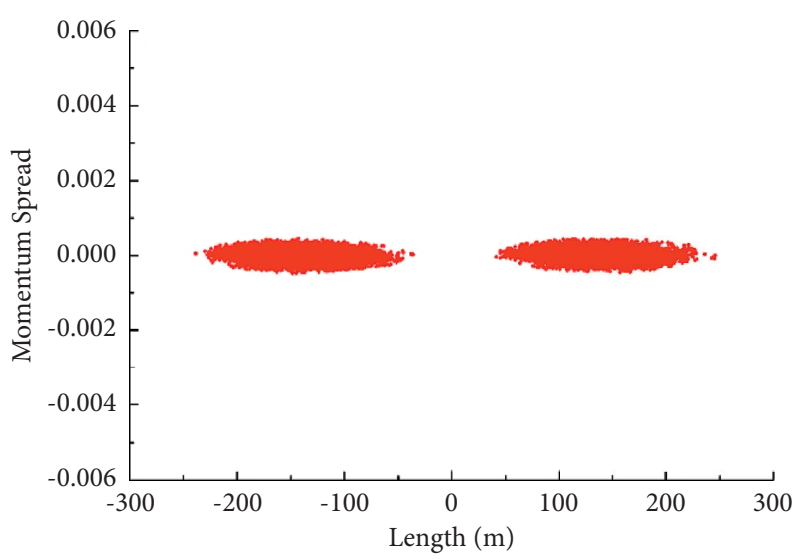

(a)

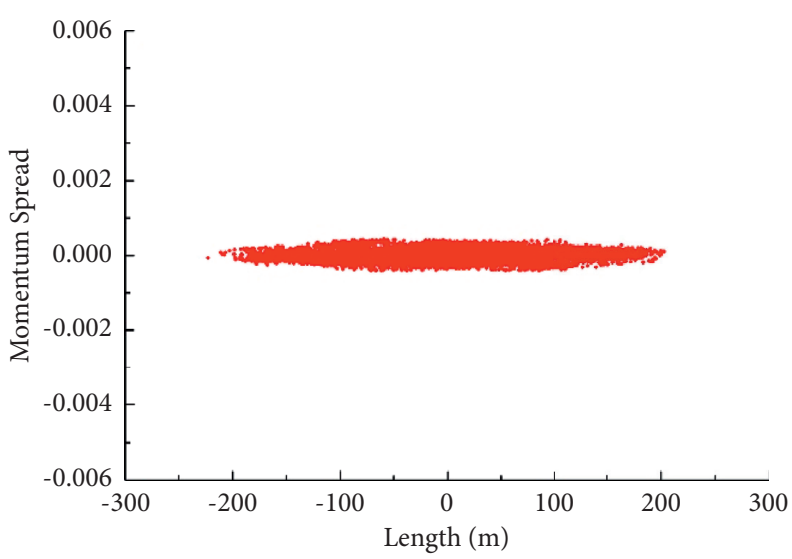

(b)

Figure 8: Beam ion distributions in the phase space at the end of the $4: 2$ bunch merging (a) and the $2: 1$ bunch merging (b).

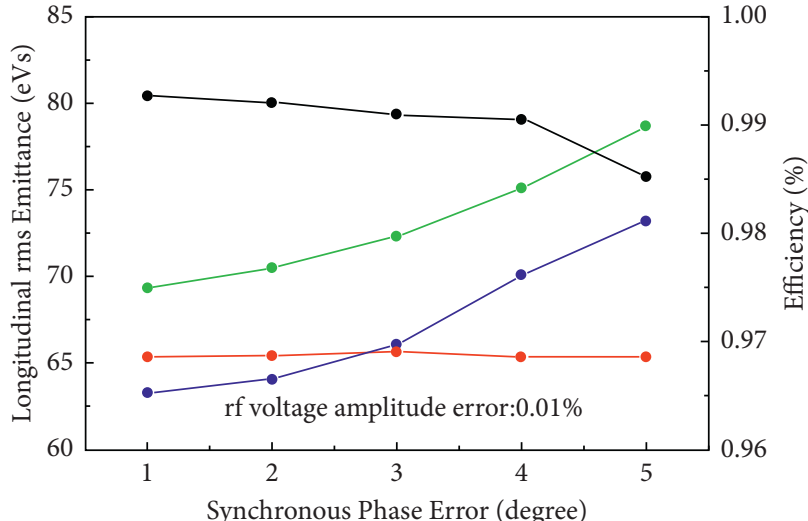

$-\bullet-$ at the end capture $\quad-\bullet$ one bunch

$-\bullet$ two bunches $\quad-\bullet$ Efficiency

(a)

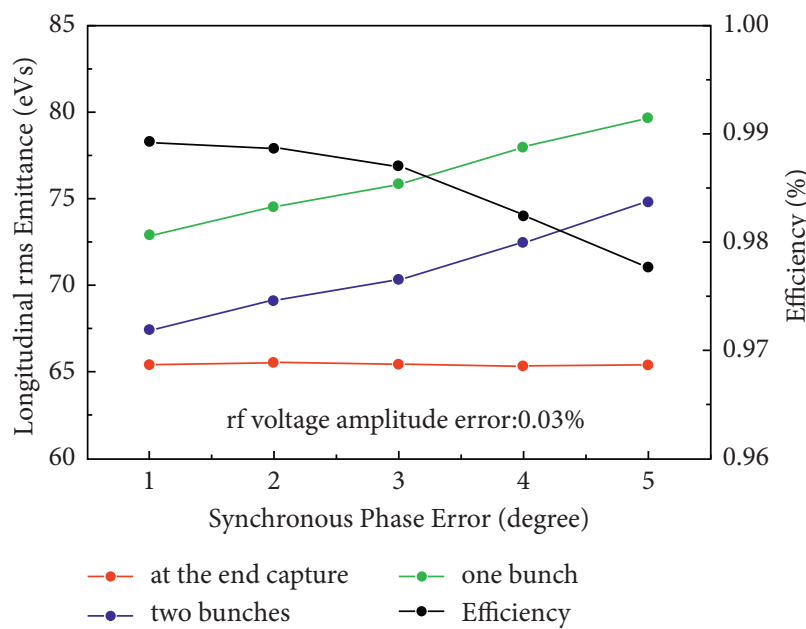

(c)

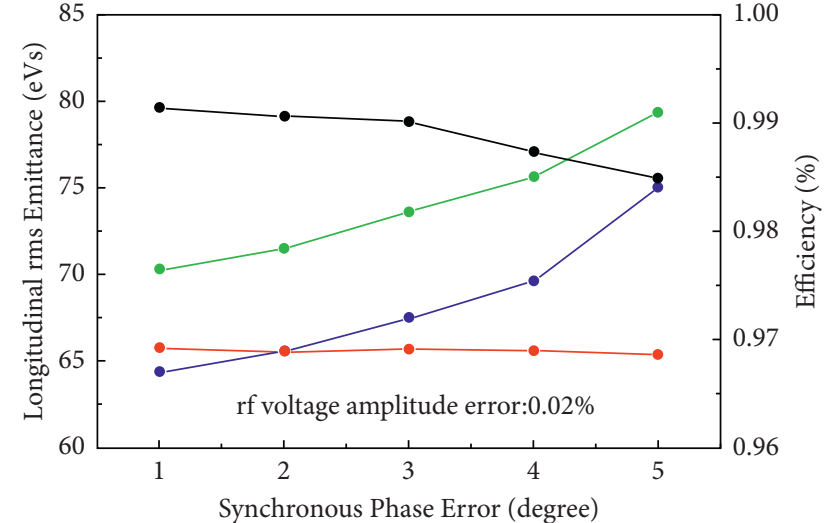

$\quad-\bullet$ at the end capture $\quad-\bullet$ one bunch

$\rightarrow-$ two bunches $\quad-\bullet$ Efficiency

(b)

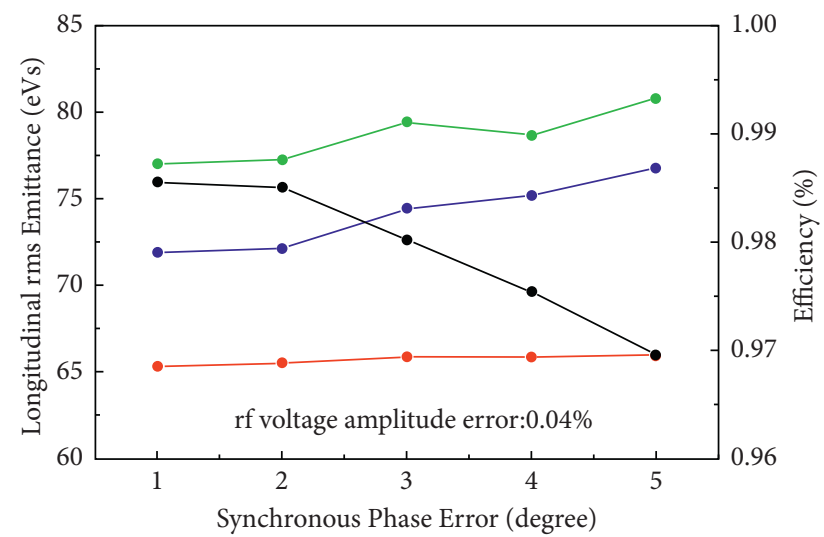

- - at the end capture $\quad \ldots-$ one bunch

- - two bunches $\quad-\bullet$ Efficiency

(d)

Figure 9: $\varepsilon_{\text {rms }}$ and efficiency vs. RF field errors in phase $\left(\Delta \varphi=1^{\circ}, 2^{\circ}, 3^{\circ}, 4^{\circ}\right.$, and $\left.5^{\circ}\right)$ and in amplitude $(\Delta V / V):($ a) $\Delta V / V=0.01 \%$; (b) $\Delta V / V=0.02 \%$; (c) $\Delta V / V=0.03 \%$; (d) $\Delta V / V=0.04 \%$. 


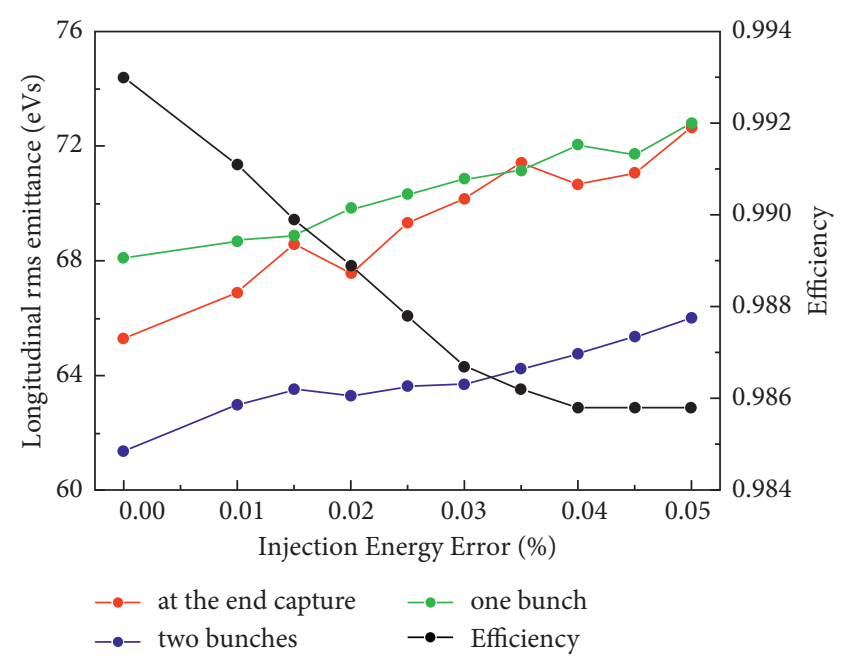

Figure 10: $\varepsilon_{\text {rms }}$ and efficiency vs. $\Delta E / E=0.01,0.02,0.03,0.04$, and 0.05 .

increases with the increase of $V_{3}$, so the RF program of $t_{2}=0.03 \mathrm{~s}$ and $V_{3}=1 \mathrm{kV}$ is determined, the phase $\varphi_{s}$ is fixed at $0.785 \mathrm{rad}$, the beam distribution in the longitudinal phase space is shown in Figure 8(b), and $\varepsilon_{\mathrm{rms}}$ is $60.19 \mathrm{eVs}$.

\section{Conclusion}

The whole process for ${ }^{238} \mathrm{U}^{35+}$ from beam capture to bunch merging takes about $0.24 \mathrm{~s}$, and the total beam loss does not exceed $1 \%$. The RF acceleration system is designed with a total peak voltage of about $268 \mathrm{kV}$, composed of $7 \mathrm{RF}$ cavities, and the frequency range is from $0.39 \mathrm{MHz}$ to $1.79 \mathrm{MHz}$.

The beam parameters obtained in this study will not only provide the basis for the design of the extraction elements of the BRing, such as kicker and electrostatic septum, but also for the injection element of the SRing.

\section{Appendix}

\section{A. Effect on Beam Dynamics with RF Errors}

The longitudinal beam manipulation can be done by the RF cavity, the calculated RF program due to the idealized RF character differs from the operating conditions, and many inevitable errors such as RF phase and amplitude field will lead to the change of the beam distribution in the phase space. In this paper, further studies were performed by using the numerical simulations to estimate the impact of RF phase and amplitude field errors on the beam longitudinal emittance growth and possible resulting beam loss by CISP. The results in turn dictate the tolerances on these errors.

The simulations, with different synchronous random phase errors ranging from $\Delta \varphi=1^{\circ}$ to $5^{\circ}$ with a step of 1 at different random amplitude errors $\Delta V / V=0.01 \%, 0.02 \%$, $0.03 \%$, and $0.04 \%$, are performed, and $\varepsilon_{\text {rms }}$ at the end of capture (red solid line), at the end of the $4: 2$ bunch merging (blue solid line), and at the end of the $2: 1$ bunch merging (green solid line) and the total efficiency (black solid line) are derived, which are shown in Figure 9.

The simulation results show that the difference of the $\varepsilon_{\text {rms }}$ growth at the end of capture is only $1.00 \%$ between the cases with the RF field errors $\Delta \varphi=5^{\circ}$ and $\Delta V / V=$ $0.04 \%$ than the idealized RF program, but the difference of the $\varepsilon_{\text {rms }}$ growth at the end of the $4: 2$ bunch merging and at the end of the $2: 1$ bunch merging is $21.12 \%$ and $16.52 \%$ with the same RF field error mentioned above; meanwhile, the total efficiency can only reach $96.97 \%$, which is far beyond the design requirement. To ensure the total beam loss below 1\%, the combined RF field errors of $\Delta \varphi \leq 2^{\circ}$ in phase and $\Delta V / V=0.02 \%$ in amplitude are needed.

\section{B. Effect on Beam Dynamics with Energy Fluctuation}

The injected energy fluctuation $\Delta E$ during the two-plane painting injection will lead to the mismatch to the synchronous energy of the BRing, which will cause the increase of energy spread, and then beam loss may occur due to the insufficient bucket area when the calculated RF program is ramped. Effects of $\Delta E / E$ on the emittance growth and beam loss were numerically investigated by CISP, and the results are shown in Figure 10.

The injected beam energy fluctuation has a great influence on $\varepsilon_{\text {rms }}$ not only after capture but also after the $4: 2$ bunch merging and the $2: 1$ bunch merging, and it also has a great impact on efficiency. To ensure the efficiency is not less than $99 \%, \Delta E / E$ shall not exceed $0.01 \%$.

\section{Data Availability}

The data used to support the findings of this study are available from the corresponding author upon request.

\section{Conflicts of Interest}

The authors declare that they have no conflicts of interest.

\section{References}

[1] X. J. Wen, W. L. Zhan, B. W. Wei et al., "HIRFL-CSR complex," Chinese Physics, vol. c33, no. 8, pp. 804-810, 2009.

[2] X. J. Wen, W. L. Zhan, B. W. Wei et al., "The heavy ion cooler storage ring project (HIRFL-CSR) at Lanzhou," Nuclear Instruments and Methods in Physics Research, Section A, vol. 488, no. 1-2, pp. 11-25, 2002.

[3] X. L. Tu, H. S. Xu, M. Wang et al., "Direct mass measurements of short-lived $\mathrm{A}=2 \mathrm{Z}-1$ nuclides ${ }^{63} \mathrm{Ge},{ }^{65} \mathrm{As},{ }^{67} \mathrm{Se}$, and ${ }^{71} \mathrm{Kr}$ and their impact on nucleosynthesis in the rp process," Physical Review Letters, vol. 106, no. 11, Article ID 112501, 2011.

[4] Y. H. Zhang, H. S. Xu, Y. A. Litvinov et al., "Mass Measurements of the Neutron-Deficient ${ }^{41} \mathrm{Ti},{ }^{45} \mathrm{Cr},{ }^{49} \mathrm{Fe}$, and ${ }^{53} \mathrm{Ni}$ Nuclides: First Test of the Isobaric Multiplet Mass Equation in fp-Shell Nuclei," Physical Review Letters, vol. 109, no. 10, Article ID 102501, 2012.

[5] X. Xu, P. Zhang, P. Shuai et al., "Identification of the Lowest $T=2, J^{\pi}=0^{+}$Isobaric Analog State in ${ }^{52}$ Co and Its Impact on 
the Understanding of $\beta$-Decay Properties of 52 ," Physical Review Letters, vol. 117, no. 18, Article ID 182503, 2016.

[6] Z. K. Huang, W. Q. Wen, S. X. Wang et al., "Absolute rate coefficients for dielectronic recombination of Na-like $\mathrm{Kr}^{25+}$," Plastics and Rubber Asia, vol. 102, Article ID 062823, 2020.

[7] Y. Z. Sun, S. T. Wang, Z. Y. Sun et al., "Two-neutron removal cross sections from ${ }^{15,16} \mathrm{C}$ at around $240 \mathrm{MeV} /$ nucleon," Physical Review C, vol. 99, Article ID 024605, 2019.

[8] Y.X. Zhao, Y.Z. Sun, S. T. Wang et al., "One-proton knockout from ${ }^{16} \mathrm{C}$ at around $240 \mathrm{MeV} /$ nucleon," Physical Review C, vol. 100, Article ID 044609, 2019.

[9] Y. J. Yuan, D. Q. Gao, L. Z. Ma et al., "Present status of HIRFL complex in Lanzhou," Journal of Physics: Conference Series, vol. 1401, Article ID 012003, 2020.

[10] J. C. Yang, J. W. Xia, G. Q. Xiao et al., "High intensity heavy ion accelerator facility (HIAF) in China," Nuclear Instruments and Methods in Physics Research Section B: Beam Interactions with Materials and Atoms, vol. 317, pp. 263-265, 2013.

[11] H. W. Zhao, L. T. Sun, J. W. Guo et al., "Superconducting ECR ion source: from 24-28 GHz SECRAL to $45 \mathrm{GHz}$ fourth generation ECR," Review of Scientific Instruments, vol. 89, no. 5, Article ID 052301, 2018.

[12] Z. J. Wang, Y. He, H. Jia et al., "Conceptual design of superconducting heavy ion linear injector for hiaf," in Proceedings of the 26th International Linear Accelerator Conference, pp. 561-563, Tel Aviv, Israel, September 2012.

[13] B. Wu, J. C. Yang, J. W. Xia et al., "The design of the spectrometer ring at the HIAF," Nuclear Instruments and Methods in Physics Research Section A: Accelerators, Spectrometers, Detectors and Associated Equipment, vol. 881, pp. 27-35, 2018.

[14] L. N. Sheng, X. H. Zhang, J. Q. Zhang et al., "Ion-optical design of high energy FRagment separator (HFRS) at HIAF," Nuclear Instruments and Methods in Physics Research Section B: Beam Interactions with Materials and Atoms, vol. 469, pp. 1-9, 2020.

[15] Y. L. Ye, X. F. Yang, L. Yang, and J. X. Han, "Radioactive ion beam physics related to HIAF," Scientia Sinica Physica, Mechanica and Astronomica, vol. 50, Article ID 112003, 2020.

[16] X.H. Zhou, Z.Y. Zhang, Z.G. Gan, and F. R. Xu, S. G. Zhou, Research program of superheavy elements and nuclides based on HIAF," Scientia Sinica Physica, Mechanica and Astronomica, vol. 50, Article ID 112002, 2020.

[17] B. Guo, W.P. Liu, X.D. Tang, Z. H. Li, and J. J. He, "Research program of nuclear astrophysics based on the HIAF," Scientia Sinica Physica, Mechanica and Astronomica, vol. 50, Article ID 112007, 2020.

[18] Y. T. Zhao, Z. M. Zhang, R. Cheng et al., "High-energy-density physics based on HIAF," Scientia Sinica Physica, Mechanica and Astronomica, vol. 50, Article ID 112004, 2020.

[19] X. W. Ma, S. F. Zhang, W. Q. Wen et al., Z. K. Huang, Atomic physics at the HIAF under extreme conditions: QED effects from strong Coulomb fields to beyond Schwinger limits," Scientia Sinica Physica, Mechanica andAstronomica, vol. 50, Article ID 112008, 2020.

[20] J. Liu, J. C. Yang, J. W. Xia et al., "CISP: simulation Platform for collective instabilities in the BRing of HIAF project," Nuclear Instruments and Methods in Physics Research Section A: Accelerators, Spectrometers, Detectors and Associated Equipment, vol. 881, pp. 36-43, 2018.

[21] S. Y. Lee, Accelerator Physics, World Scientific Publishing, Singapore, 1999.

[22] L. Liu, J. Y. Tang, J. Qiu, and T. Wei, "Longitudinal RF capture and acceleration simulation in CSNS RCS," Chinese Physics C, vol. 33, no. Suppl.II, pp. 4-7, 2009.
[23] A. V. Eliseev, I. N. Meshkov, V. A. Mikhailov, and A. O. Sidorin, "Longitudinal dynamics of 197Au32+ and 197Au79+ ions in NICA injection chain," Physics of Particles and Nuclei Letters, vol. 7, no. 7, pp. 473-477, 2010.

[24] O. Boine-Frankenheim, "Space charge effects in bunches for different rf wave forms," Physical Review Special Topics Accelerators and Beams, vol. 8, Article ID 034201, 2005.

[25] P. J. Spiller, K. Blasche, P. Hülsmann, A. Krämer, H. Ramakers, and H. Reich-Sprenger, "High intensity uranium operation in SIS18," in Proceedings of the 9th European Particle Accelerator Conference (EPAC 2004), pp. 1180-1182, Lucerne, Switzerland, July 2004.

[26] L. Rybarcyk, "Longitudinal tracking studies for the AHF booster synchrotron," in Proceedings of the 2003 Particle Accelerator Conference, pp. 1566-1568, Portland, OR, USA, May 2003.

[27] H. Hotchi, M. Kinsho, K. Hasegawa et al., "Beam commissioning of the $3-\mathrm{GeV}$ rapid cycling synchrotron of the Japan proton accelerator research complex," Physical Review Special Topics-Accelerators and Beams, vol. 12, Article ID 040402, 2009.

[28] B. Zipfel, H. Klingbeil, U. Laier, K.-P. Ningel, and C. Thielmann, 4:2:1 Bunch Merging in SIS18, GSI Scientific Reports, Kolkata, India, 2010.

[29] I. Bozsik, I. Hofmann, and A. Jahnke, "Numerical investigation of bunch merging in a heavy ion synchrotron," in Proceedings of the Computing in Accelerator Design and Operation, pp. 128-133, Berlin, Germany, September 1983. 